\title{
Strongly modulated transmission of a spin-split quantum wire with local Rashba interaction
}

\author{
David Sánchez, ${ }^{1}$ Llorenç Serra, ${ }^{1,2}$ and Mahn-Soo $\mathrm{Choi}^{3}$ \\ ${ }^{1}$ Departament de Física, Universitat de les Illes Balears, E-07122 Palma de Mallorca, Spain \\ ${ }^{2}$ Institut Mediterrani d'Estudis Avançats IMEDEA (CSIC-UIB), E-07122 Palma de Mallorca, Spain \\ ${ }^{3}$ Department of Physics, Korea University, Seoul 136-701, Korea \\ (Received 4 June 2007; revised manuscript received 13 September 2007; published 14 January 2008)
}

\begin{abstract}
We investigate the transport properties of ballistic quantum wires in the presence of Zeeman spin splittings and a spatially inhomogeneous Rashba interaction. The Zeeman interaction is extended along the wire and produces gaps in the energy spectrum, which allow electron propagation only for spinors lying along a certain direction. For spins in the opposite direction, the waves are evanescent far away from the Rashba region, which plays the role of the scattering center. The most interesting case occurs when the magnetic field is perpendicular to the Rashba field. Then, the spins of the asymptotic wave functions are not eigenfunctions of the Rashba Hamiltonian, and the resulting coupling between spins in the Rashba region gives rise to sudden changes of the transmission probability when the Fermi energy is swept along the gap. After briefly examining the energy spectrum and eigenfunctions of a wire with extended Rashba coupling, we analyze the transmission through a region of localized Rashba interaction, in which a double interface separates a region of constant Rashba interaction from wire leads free from spin-orbit coupling. For energies slightly above the propagation threshold, we find the ubiquitous occurrence of transmission zeros (antiresonances), which are analyzed by matching methods in the one-dimensional limit. We find that a minimal tight-binding model yields analytical transmission line shapes of Fano antiresonance type. The general angular dependence of the external magnetic field is treated within projected Schrödinger equations with the nondiagonal Hamiltonian matrix elements mixing different wave function components. Finally, we consider a realistic quantum wire where the energy subbands are coupled via the Rashba intersubband coupling term and discuss its effect on the transmission zeros. We find that the antiresonances are robust against intersubband mixing, magnetic field changes, and smooth variations of the wire interfaces, which paves the way for possible applications of spin-split Rashba wires as spintronic current modulators.
\end{abstract}

DOI: 10.1103/PhysRevB.77.035315

PACS number(s): 73.63.Nm, 71.70.Ej, 72.25.Dc

\section{INTRODUCTION}

\section{A. Motivation}

Since the pioneering Datta-Das proposal of an electronic field-effect transistor in which the current flow is controlled by magnetic means only, ${ }^{1}$ the study of the Rashba ${ }^{2,3}$ spinorbit interaction in one-dimensional (1D) and quasi-onedimensional ballistic channels (quantum wires) has attracted a lot of interest. ${ }^{4-35}$ Precise tunability of the strength of the Rashba coupling has also been experimentally demonstrated in quantum wells. ${ }^{36-38}$ Typically, semiconductor quantum wires are built from two-dimensional electron gases formed at the interface of a semiconductor heterostructure when the lateral motion of electrons is restricted by a transversal confinement potential to effective widths of the order of the de Broglie electron wavelength. For very clean quantum wires (e.g., quantum point contacts), transport is ballistic and conductance is quantized to integer values of $e^{2} / h \cdot{ }^{39,40}$

The presence of impurities or defects in the vicinity of the constriction destroys conductance quantization. ${ }^{41-46}$ A striking effect arises when the impurity potential is attractive and enables the existence of at least one bound state whose energy is degenerate with the continuum band of propagating states. As a consequence, for energies close to the transition threshold, a direct transmission channel can interfere with a wave trajectory that travels across the bound state, and this interference is destructive, leading to enhanced backscattering and Fano asymmetric line shapes. ${ }^{47-50}$ Recently, two of us $^{30}$ have demonstrated that a spin-orbit interaction of the Rashba type localized in an infinitely long quantum wire plays a role similar to an attractive potential, and pronounced dips are seen in numerical simulations of the conductance curves. ${ }^{20,27,30,33}$ It is remarkable that the Rashba interaction provides both the attractive potential that supports bound states $^{51,52}$ and the mixing term that couples the localized and the propagating states. ${ }^{30}$ Interestingly, when charging effects are taken into account, Coulomb blockade resonances can be tuned, directly modulating the strength of the Rashba coupling. ${ }^{53}$

A magnetic field applied in the wire plane leads to Zeeman spin splitting of the 1D modes. Evidence of this is seen in the appearance of conductance plateaus at odd multiples of $e^{2} / h{ }^{40}$ In the first plateau, the current is fully polarized since only one spin species is allowed to propagate. Quantum states with opposite spins are evanescent asymptotically and do not take part in electron transport unless there exist inhomogeneities that give rise to resonances or Fano-type interferences, in which case evanescent states are crucial. Reference 54 presents a theoretical method to calculate evanescent states in quantum wires with uniform Rashba interaction.

To determine the full transmission pattern of a generic quantum wire, one must first analyze the energy spectrum of the wire. For quantum wires with uniform Rashba interaction in the absence of external magnetic fields, free-electron energy bands are parabolas shifted apart for opposite spin directions. ${ }^{8}$ The splitting size is proportional to the spin-orbit 
interaction strength $\alpha$, and in the quasi-1D case the Rashba interaction produces anticrossings between bands corresponding to opposite spins and adjacent modes. ${ }^{5,10}$ Moreover, the propagation threshold is shifted, compared to the case with no spin-orbit coupling, down by an energy of $m \alpha^{2} / 2 \hbar^{2}$. In the presence of an in-plane magnetic field, the energy spectrum changes dramatically even for arbitrarily small fields. The field can be either externally applied or originated from stray fields of the ferromagnets coupled to the wire in the Datta-Das setup. ${ }^{1}$ It is shown ${ }^{14,16,26}$ that the interplay between the magnetic field and the Rashba interaction leads to the openings of gaps in the 1D energy bands at small wave numbers. In a quasi-1D wire, most of the energy dispersions around the gap form energy minima locally in contrast to the maxima encountered in the $1 \mathrm{D}$ case. ${ }^{26}$ In those energy windows, in which the gap consists of an energy local maximum followed by a local minimum, the conductance curves present anomalous steps for Fermi energies within the gap. ${ }^{16,26}$

In short, external magnetic fields lead to the formation of energy gaps in the spectrum, while local Rashba interactions produce Fano-type antiresonances due to the formation of quasibound states coupled to the channel of direct transmission states. Therefore, we expect a rich interplay between in-plane fields and localized Rashba spin-orbit couplings in the transport properties of a ballistic quantum wire. This paper presents a generic theoretical description of the quantum transmission of an electron subject to Zeeman splittings and spatially modulated Rashba fields.

\section{B. Main findings}

We find the occurrence of exact transmission zeros in the conductance curves of a Zeeman-split wire with local Rashba interaction as a function of the Fermi energy. Central to the existence of the transmission zeros are the formation of a Zeeman gap arising from an in-plane magnetic field and the role of the evanescent states within the Rashba region. In fact, the Rashba interaction couples the propagating and evanescent states precisely in the interior of the Rashba region. The transmission antiresonances are almost universal, showing a vanishingly small transmission at moderately low magnetic fields. This might be relevant for applications since it provides two operation points for working transistors (low and high current states). It is important to stress that these transmission zeros are fundamentally distinct from the suppressed transmission that may take place in a Datta-Das setup due to spin precession ${ }^{1}$ even in the presence of in-plane magnetic fields. ${ }^{35}$ The antiresonance position can be tuned with a slight change of $\alpha$ and is robust against changes of the magnetic field. We only require that the Fermi energy lies within the gap.

\section{Outline}

The outline of the paper is as follows. Section II is devoted to analyzing the transport properties of a 1D wire, where only one subband is taken into account, and Rashbainduced intersubband coupling is then neglected. In Sec. II A, we discuss the eigenstates and energy spectrum of a 1D wire subject to Rashba interaction and Zeeman spin splittings. In Sec. II B, we consider a finite Rashba region with constant Rashba strength and a magnetic field pointing in a direction perpendicular to the Rashba field and we calculate the transmission within the scattering formalism and numerical matching. A tight-binding description of the problem is considered in Sec. II C. We derive an exact expression for the transmission in the limit of a minimal Rashba region and discuss the Fano form of the line shape. To end Sec. II, we present in Sec. II D results for the angular dependence of the magnetic field direction. In Sec. III, we examine a quasi-1D wire. The numerical results are in agreement with the 1D case, thus demonstrating that the sharp antiresonances are robust even when intersubband coupling is present like in realistic wires. We also discuss the case of an arbitrary dependence of the Rashba strength with the position and compare our results when the Rashba interaction smoothly increases at the interfaces. Finally, Sec. IV contains the conclusions.

\section{ONE-DIMENSIONAL WIRE}

We consider a two-dimensional (2D) electron gas formed in the $x-y$ plane due to a strong confinement in the $z$ direction. As a result of the interfacial electric field, there arises a spin-orbit coupling of the Rashba type with a Hamiltonian given by

$$
\mathcal{H}_{R}=\frac{\alpha(x)}{2 \hbar}\left(p_{y} \sigma_{x}-p_{x} \sigma_{y}\right)+\text { H.c. },
$$

where the Rashba strength $\alpha(x)$ can be spatially modulated. The limit of a purely 1D system is obtained by further constraining the electron motion along, e.g., the $x$ direction. Then, the $p_{y} \sigma_{x}$ term in Eq. (1) is neglected and the Rashba interaction plays the effective role of a momentumdependent magnetic field with a direction along the $y$ axis. In the following section, we discuss the spectrum and eigenfunctions of a 1D wire when the Rashba strength is uniform and the external magnetic field points either in the $x-y$ plane ("in-plane" field) or along the vertical $z$ direction ("perpendicular" field).

\section{A. Extended Rashba interaction}

\section{In-plane field}

We consider an in-plane magnetic field with arbitrary direction, $\vec{B}=(B \cos \theta, B \sin \theta, 0)$, giving rise to a Zeeman interaction. ${ }^{16,26}$ Then, the single-particle Hamiltonian reads

$$
\begin{aligned}
\mathcal{H}_{1 \mathrm{D}}= & \frac{-\hbar^{2}}{2 m} \frac{d^{2}}{d x^{2}}+\frac{\Delta_{Z}}{2}\left(\sigma_{x} \cos \theta+\sigma_{y} \sin \theta\right) \\
& -\frac{1}{2 \hbar} \sigma_{y}\left\{\alpha(x),-i \hbar \frac{d}{d x}\right\},
\end{aligned}
$$

where $\Delta_{Z}=g \mu_{B} B$ is the Zeeman splitting. When we assume a constant Rashba strength $\alpha(x) \equiv \alpha=$ const from $-\infty$ to $\infty$, the Hamiltonian $\mathcal{H}_{1 \mathrm{D}}$ is diagonalized using the spinor wave functions (we take the spin quantization direction along $z$ ), 


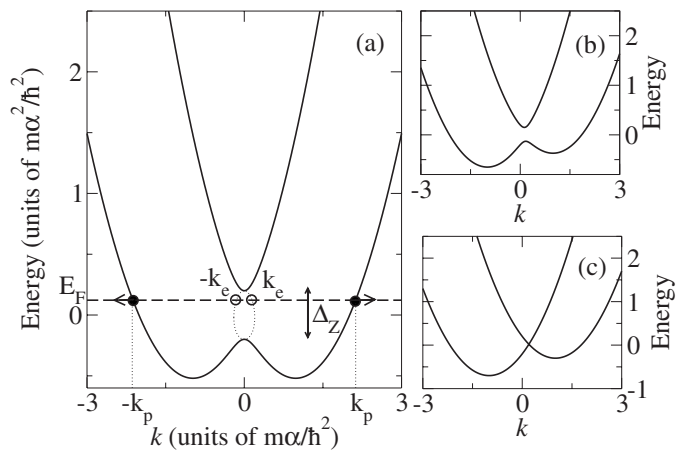

FIG. 1. Energy spectrum for a 1D quantum wire with uniform Rashba coupling for a Zeeman splitting $\Delta_{Z}=0.4 m \alpha^{2} / \hbar^{2}$ and different magnetic field angles: (a) $\theta=0$, (b) $\theta=\pi / 4$, and (c) $\theta=\pi / 2$.

$$
\psi_{\eta}(x)=\frac{1}{\sqrt{2}}\left(\begin{array}{c}
e^{i \Omega_{k} / 2} \\
\eta e^{-i \Omega_{k} / 2}
\end{array}\right) e^{i k x}
$$

where $\eta= \pm$ is the branch-splitting quantum index and $k$ is the wave vector associated with free motion along $x$. The spin orientation is determined from

$$
\tan \Omega_{k}(\theta)=\frac{\alpha k-\left(\Delta_{Z} / 2\right) \sin \theta}{\left(\Delta_{Z} / 2\right) \cos \theta} .
$$

We note that there exists no common spin quantization axis since $\Omega_{k}$ depends on $k{ }^{26}$ This is due to the existence of a magnetic field since for $\Delta_{Z}=0$ the spinors lie along $y$ (the Rashba axis). The effect is akin to a $2 \mathrm{D}$ system with Rashba interaction for which $\tan \Omega=k_{x} / k_{y}$. However, in the $2 \mathrm{D}$ case the spin orientation is always perpendicular to $\vec{k}$ whereas in the 1D case only for asymptotically large momenta $(|k|$ $\rightarrow \infty$ ) the spin is quantized along $y$. A similar effect arises from Rashba intersubband coupling in quasi-1D systems. ${ }^{10}$

From the Schrödinger equation, $\mathcal{H}_{1 \mathrm{D}} \psi_{\eta}=E_{\eta} \psi_{\eta}$, one finds the energy spectrum,

$$
E_{\eta}(k)=\frac{\hbar^{2} k^{2}}{2 m}+\eta \sqrt{\alpha^{2} k^{2}+\left(\Delta_{Z} / 2\right)^{2}-\alpha k \Delta_{Z} \sin \theta} .
$$

For $\theta=0$, the case of interest occurs for small Zeeman splittings, $\Delta_{Z}<2 m \alpha^{2} / \hbar^{2}$ [see Fig. 1(a)]. This gives the condition for the opening of a pseudogap region for which the lowest branch of the spectrum develops a local maximum around $k=0$. This fact has important consequences for the electronic transport. ${ }^{14,16,17,26}$ (Larger magnetic fields, $\Delta_{Z}>2 m \alpha^{2} / \hbar^{2}$, turn the local maximum into a local minimum, but this case is less interesting and will not be treated in this work.) For Fermi energies lying in the pseudogap region, $-\Delta_{Z} / 2<E_{F}$ $<\Delta_{Z} / 2$, there is only a wave function with a given spin direction for each mover (right moving or left moving). In addition, there also exist evanescent waves, which are crucial when the Rashba interaction is confined to a finite region. Outside the pseudogap region, there are four real wave vectors for a given $E_{F}$.
Increasing $\theta$ from 0 to $\pi / 2$ leads to the progressive reduction of the pseudogap size [see Figs. 1(b) and 1(c)]. For $\theta=\pi / 2$, the gap vanishes and the spinors point along the $y$ axis since in this case the field axis and the Rashba axis coincide.

We note in passing that when $\alpha(x)$ is constant (or, more generically, an even function of $x$ ), there exists a symmetry property of the Hamiltonian given by Eq. (2). Let us concentrate on the case $\theta=0$. Thus, $\mathcal{H}_{1 \mathrm{D}}$ is invariant under the transformation

$$
\hat{Z}=i \Pi \exp \left[-i \pi \hat{\sigma}_{x}\right]=\Pi \hat{\sigma}_{x},
$$

namely, the rotation by $\pi$ around the $x$ axis in the spin space followed by the parity operator $\Pi$, which yields inversion in the $x$ direction. The additional factor of $i$ is to ensure that $\hat{Z}^{2}=1$. Similar symmetry properties have been discussed in Refs. 7, 25, and 55, which find that the spin parity, i.e., the combination of parity and a Pauli matrix, is a constant of motion for $B=0$. Here, $\Pi \hat{\sigma}_{x}$ commutes with $\mathcal{H}_{1 \mathrm{D}}$ even for nonzero fields when $\theta=0$. Hence, one can find a common basis of eigenstates for $\mathcal{H}_{1 \mathrm{D}}$ and $\hat{Z}$. The wave functions given by Eq. (3) are not eigenstates of $\hat{Z}$. In fact, $\left|\psi_{\eta,+|k|}\right\rangle$ $=\eta \hat{Z}\left|\psi_{\eta,-|k|}\right\rangle$ since $\Omega \rightarrow-\Omega$ when $|k| \rightarrow-|k|$. Therefore, one could construct states with definite parities with regard to $\hat{Z}$ from even (e) and odd $(o)$ combinations of $\left|\psi_{\eta, \pm|k|}\right\rangle$, i.e., $\left|\psi_{\eta}^{e / o}\right\rangle=\left|\psi_{\eta,+|k|}\right\rangle \pm \eta\left|\psi_{\eta,-|k|}\right\rangle$.

\section{Perpendicular field}

For fields pointing along $z, \vec{B}=(0,0, B)$, the Zeeman term in Eq. (2) is expressed as $\left(\Delta_{Z} / 2\right) \sigma_{z}$. The spectrum is identical to the $\theta=0$ case [Fig. 1(a)]. Therefore, for the sake of the present discussion, the cases $\vec{B}$ parallel to $z$ and $\vec{B}$ parallel to $x$ are equivalent in the $1 \mathrm{D}$ case since both are perpendicular to the Rashba field direction (along $y$ ). Of course, in the quasi-1D case, one should also take into account orbital effects, but in this section we can neglect this. Let us focus on the $\eta=-$ branch and energies within the pseudogap. We will find that strong transmission changes take place in that region.

For $-\Delta_{Z} / 2<E_{F}<\Delta_{Z} / 2$, there are two propagating solutions,

$$
\psi(x)=\left(\begin{array}{c} 
\pm \sin \frac{\Omega_{p}}{2} \\
i \cos \frac{\Omega_{p}}{2}
\end{array}\right) e^{ \pm i k_{p} x},
$$

with wave vector [see Fig. 1(a)]

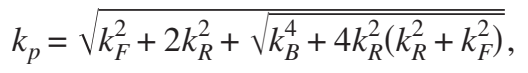

where we have defined

$$
\begin{gathered}
k_{F}=\sqrt{2 m E_{F} / \hbar^{2}}, \\
k_{R}=m \alpha / \hbar^{2},
\end{gathered}
$$




$$
k_{B}=\sqrt{m \Delta_{Z} / \hbar^{2}} .
$$

As discussed above, the angle $\Omega_{p}$ depends on the wave vector,

$$
\tan \Omega_{p}=\frac{2 k_{R} k_{p}}{k_{B}^{2}} .
$$

Further, the pseudogap region admits two more solutions, which are evanescent waves. For $E_{F}<-\hbar^{2} B^{2} / 2 m \alpha^{2}$ (i.e., $\left.k_{F}^{2}<k_{B}^{4} / 4 k_{R}^{2}\right)$, we find

$$
\psi(x)=\left(\begin{array}{c}
\mp \sinh \frac{\Omega_{e}}{2} \\
\cosh \frac{\Omega_{e}}{2}
\end{array}\right) e^{ \pm k_{e} x} .
$$

For $E_{F}>-\hbar^{2} B^{2} / 2 m \alpha^{2}$, one must make the replacements $\sinh \rightarrow \cosh$ and $\cosh \rightarrow \sinh$. Moreover,

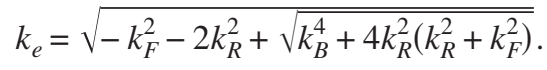

and

$$
\tanh \Omega_{e}=\frac{2 k_{R} k_{e}}{k_{B}^{2}} .
$$

In Fig. 1(a), we illustrate the "dispersion" relation for the evanescent states and the location of $\pm k_{e}$. The evanescent states make sense only for nonvanishing $\Delta_{Z}$, as can be readily seen by setting $k_{B}=0$ in Eqs. (8) and (14). $k_{p}$ becomes $k_{R}+\sqrt{k_{R}^{2}+k_{F}^{2}}$ and $k_{e}$ becomes purely imaginary, $k_{e}=i\left(k_{R}\right.$ $\left.-\sqrt{k_{R}^{2}+k_{F}^{2}}\right)$. This corresponds to four propagating solutions

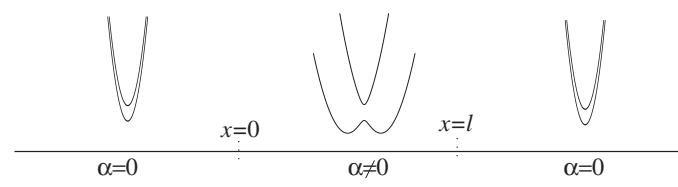

FIG. 2. Schematic representation of a local Rashba interaction in a $1 \mathrm{D}$ quantum wire.

(two left moving and two right moving) with a definite spin direction. $^{8}$

\section{B. Local Rashba interaction}

We now consider a double interface at $x=0$ and $x=l$ between a normal conduction band $(x<0$ and $x>l)$ and a region of localized Rashba interaction extending from $x=0$ to $x=l$ (see Fig. 2). Then, $\alpha(x) \equiv \alpha=$ const for $0<x<l$ and $\alpha(x)=0$ elsewhere. Our basic goal is to find the transmission $T$ through the Rashba region when the magnetic field is present all along the wire, producing a Zeeman gap. For convenience, we take the $\vec{B}$ direction along $z$ since the solutions for $x<0$ are simpler to write down.

We are interested in energies inside the spin pseudogap, $-\Delta_{Z} / 2<E_{F}<\Delta_{Z} / 2$, for which a spin-down (spin-up) electron wave is propagating (evanescent). In the scattering problem, an electron with spin down is injected from $-\infty$ and reflected with a certain probability $R=1-T$. Since the spin quantization axis in the region $0<x<l$ depends on the wave vector, we must also take into account the spin-up evanescent waves at $x<0$ and $x>l$. As a consequence, the scattering wave function for $-\Delta_{Z} / 2<E_{F}<-\hbar^{2} B^{2} / 2 m \alpha^{2}$ reads

$$
\psi(x)= \begin{cases}\left(\begin{array}{l}
0 \\
1
\end{array}\right) e^{i k_{1} x}+\left(\begin{array}{l}
0 \\
1
\end{array}\right) A e^{-i k_{1} x}+\left(\begin{array}{l}
1 \\
0
\end{array}\right) B e^{k_{2} x}, & x<0 \\
\left(\begin{array}{l}
\sin \frac{\Omega_{p}}{2} \\
i \cos \frac{\Omega_{p}}{2}
\end{array}\right) C e^{i k_{p} x}+\left(\begin{array}{c}
-\sin \frac{\Omega_{p}}{2} \\
i \cos \frac{\Omega_{p}}{2}
\end{array}\right) D e^{-i k_{p} x}+\left(\begin{array}{c}
\sinh \frac{\Omega_{e}}{2} \\
\cosh \frac{\Omega_{e}}{2}
\end{array}\right) F e^{-k_{e} x}+\left(\begin{array}{c}
-\sinh \frac{\Omega_{e}}{2} \\
\cosh \frac{\Omega_{e}}{2}
\end{array}\right) G e^{k_{e} x}, & 0<x<l \\
\left(\begin{array}{l}
0 \\
1
\end{array}\right) J e^{i k_{1}(x-l)}+\left(\begin{array}{l}
1 \\
0
\end{array}\right) K e^{-k_{2}(x-l)}, & x>l,\end{cases}
$$

whereas for $-\hbar^{2} B^{2} / 2 m \alpha^{2}<E_{F}<\Delta_{Z} / 2$, one should make the replacements $\sinh \rightarrow \cosh$ and $\cosh \rightarrow \sinh$ in the expressions for the evanescent states of the Rashba region. In Eq. (16), the wave vector $k_{1}=\sqrt{k_{F}^{2}+k_{B}^{2}}$ is written in terms of the Fermi wave vector $k_{F}^{2}=2 m E_{F} / \hbar^{2}$ and $k_{B}^{2}=m \Delta_{Z} / \hbar^{2}$, defined as before. We note that $k_{F}$ can be purely imaginary for $E_{F}<0$, though the physical wave vector $k_{1}$ is always a quantity manifestly positive and real. The evanescent wave is described with an exponentially decreasing wave with range of $\sim 1 / k_{2}$, where $k_{2}=\sqrt{k_{B}^{2}-k_{F}^{2}}$; therefore the probability of find- ing a spin up on the left or right sides is nonzero. At small $\Delta_{Z}$, the propagating states with wave vector $\pm k_{p}$ have their spins approximately along $\pm y[\Omega \approx \pi / 2$ in Eq. (3)].

We numerically find the coefficients $A, B, C, D, F, G, J$, and $K$ from matching equations. At the interfaces, the wave function must be continuous, e.g., $\psi\left(0^{-}\right)=\psi\left(0^{+}\right)$. Moreover, the flux, given by the velocity operator $\hat{v}$, must also be continuous, i.e., $\hat{v} \psi\left(0^{-}\right)=\hat{v} \psi\left(0^{+}\right)$. On the normal sides, $\hat{v}$ is trivially given by $-i(\hbar / m) d / d x$, while in the spin-orbit region one has ${ }^{8}$ 


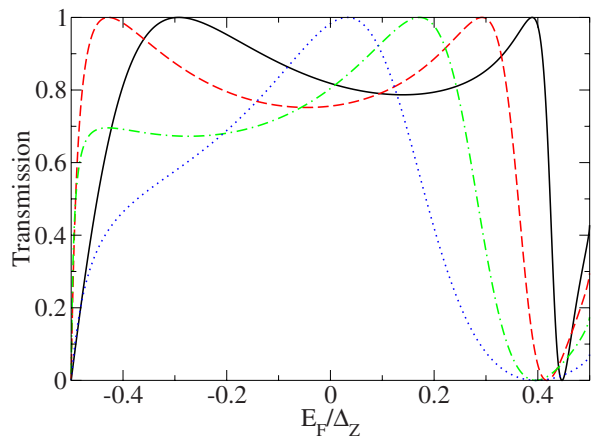

FIG. 3. (Color online) Transmission through a Rashba region for $\Delta_{Z}=12.8 \hbar^{2} / \mathrm{ml}^{2}$ and different spin-orbit intensities: $\alpha=6.4 \hbar^{2} / \mathrm{ml}$ (full line), $6.64 \hbar^{2} / \mathrm{ml}$, (dashed), $6.88 \hbar^{2} / \mathrm{ml}$, (dot-dashed), and $7.12 \hbar^{2} / m l$ (dotted).

$$
\hat{v}=\frac{i \hbar}{m}\left(\begin{array}{cc}
-\frac{d}{d x} & k_{R} \\
-k_{R} & -\frac{d}{d x}
\end{array}\right) .
$$

Importantly, the flux associated with Eq. (7), which is proportional to

$$
\langle\hat{v}\rangle= \pm k_{p}-k_{R} \sin \Omega_{p},
$$

is positive (negative) for $+k_{p}\left(-k_{p}\right)$.

The transmission and reflection probability are given by $T=|J|^{2}$ and $R=|A|^{2}$, respectively. Since we are interested in the relative influence of $\alpha$ and $\Delta_{Z}$ while keeping the Rashba region length $l$ constant, in the numerical results we give the energies as a function of the energy unit $\hbar^{2} / m l^{2}$. A characteristic transmission curve is plotted in Fig. 3 for $\Delta_{Z}$ $=12.8 \hbar^{2} / m l^{2}$, whereas $\alpha$ is slightly varied. We make the important observation that there arises an exact transmission zero near $E_{F}=\Delta_{Z} / 2$ when $\alpha=6.4 \hbar^{2} / \mathrm{ml}$. On increasing $\alpha$, the resonance position shifts to lower energies and, at the same time, the resonance broadening is enhanced. This dependence with $\alpha$ will become clear later when we discuss the tight-binding model.

The transmission for $\alpha=6.4 \hbar^{2} / m l$ and various magnetic fields is shown in Fig. 4. The transmission curves are reminiscent of Fano line shapes. An interesting question is thus whether the transmission behavior is indeed related to a Fano-type interference effect. While in strict onedimensional systems the interference giving rise to Fano line shapes is not possible due to the existence of one channel only, in this case and due to the Zeeman splitting there exist two modes, namely, the propagating mode (spin down) and the evanescent mode (spin up). Both modes become coupled locally within the Rashba region. As a result, the effect is due to a subtle combination of spin-orbit interaction and Zeeman splitting, which leads to destructive interference in the Rashba region. A simplified model, discussed below, will shed light on this. For the moment, we note that Fig. 4 shows that it is sufficient to have a rather small Zeeman gap $\Delta_{Z}^{\text {crit }}$ above which the antiresonance develops. For $\alpha=6.4 \hbar^{2} / \mathrm{ml}$, we find $\Delta_{Z}^{\text {crit }} \simeq 0.064 \hbar^{2} / m l^{2}$.

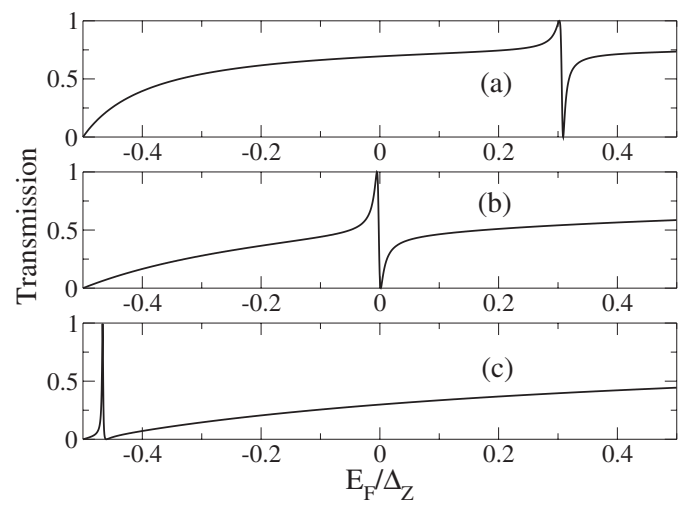

FIG. 4. Transmission versus Fermi energy for $\alpha=6.4 \hbar^{2} / \mathrm{ml}$ and different Zeeman splittings: $\Delta_{Z}=0.384 \hbar^{2} / m l^{2}$ (a), $0.128 \hbar^{2} / m l$ (b), and $0.064 \hbar^{2} / m l$ (c).

The energy and length scales we considered above are within the scope of present techniques. For example, for a Rashba region of size $l=2 \mu \mathrm{m}$, the value $\alpha=6.4 \hbar^{2} / m l$ corresponds to $\alpha \simeq 10 \mathrm{meV} \mathrm{nm}$, which is accessible in an InAs wire. ${ }^{36}$ The Zeeman energy $\Delta_{Z}=12.8 \hbar^{2} / m l^{2}$ used in Fig. 3 corresponds to a magnetic field $B \simeq 10 \mathrm{mT}$ in the same material, and $\Delta_{Z}^{\text {crit }}$ in Fig. 4(c) is only $60 \mu \mathrm{T}$. Notably, the effect scales with $l$. Therefore, a smaller $\alpha$ would require a larger wire for the antiresonance to be observable.

\section{Tight-binding model}

The continuum model discussed above leads to remarkable predictions for the conductance of a spin-split quantum wire with a local Rashba interaction, but to gain further insight it would be highly desirable to have a simplified model capable of yielding closed analytical formulas for the dip position and shape. In this subsection, we consider a discretized version of the Hamiltonian $\mathcal{H}_{1 \mathrm{D}}$ [Eq. (2)]. The infinite $1 \mathrm{D}$ wire is modeled with a linear chain of sites. Thus, we obtain the following tight-binding Hamiltonian: ${ }^{56}$

$$
\begin{aligned}
\mathcal{H}_{\mathrm{tb}}= & \sum_{i} \varepsilon_{\sigma} c_{i \sigma}^{\dagger} c_{i \sigma}-\sum_{\langle i j\rangle} t\left(c_{i \sigma}^{\dagger} c_{j \sigma}+\text { H.c. }\right) \\
& +\lambda \sum_{n}\left(c_{n \uparrow}^{\dagger} c_{(n+1) \downarrow}-c_{n \downarrow}^{\dagger} c_{(n+1) \uparrow}+\text { H.c. }\right),
\end{aligned}
$$

where the summations over $i$ and $j$ are carried out on an infinitely extended 1D wire and the last summation is over the sites of the Rashba region. In this equation, $t=\hbar^{2} / 2 m a^{2}$ couples nearest neighbors, $\lambda=\alpha / 2 a$ is the Rashba interaction strength, which couples electronic states with opposite spin directions along $z(\sigma=\{\uparrow, \downarrow\})$, and $a$ is the lattice parameter. The on-site energies are given by $\varepsilon_{\sigma}=s \Delta_{Z} / 2[s=+(-)$ for $\sigma=\uparrow(\downarrow)]$. The Hamiltonian $\mathcal{H}_{\mathrm{tb}}$ is equivalent, in the limit $a$ $\rightarrow 0$, to $\mathcal{H}_{1 \mathrm{D}}$ whose transport properties have been analyzed above. Here, in order to obtain simplified expressions, we consider a localized Rashba interaction restricted only to two sites, 0 and 1 (see Fig. 5). We note that this is the minimal model that characterizes the transport properties of a $1 \mathrm{D}$ wire with a Rashba region. 


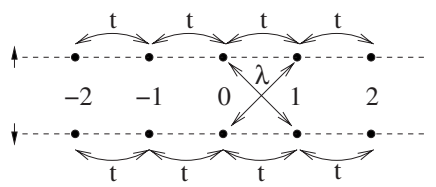

FIG. 5. In the minimal tight-binding model, the system consists of a linear chain of coupled sites with a localized Rashba interaction at sites $n=0$ and $n=1$.

For $\Delta_{Z}=0$ and $\lambda=0$, the energy band spectrum is given by the well known expression $E=-2 t \cos k a$. In the presence of a magnetic field, the spectrum becomes spin split. We now focus on an energy range close to the band bottom, $-2 t$ $-\Delta_{Z} / 2 \leqslant E \leqslant-2 t+\Delta_{Z} / 2$. Then, the eigenfunctions corresponding to spin $\downarrow(\uparrow)$ are propagating (evanescent) waves. Since we intend to solve the scattering problem of an $\downarrow$-electron wave impinging onto the Rashba region, we now introduce the wave amplitudes $\psi_{n \downarrow}=e^{i k_{\downarrow} n a}+r e^{-i k_{\downarrow} n a}$ for $n \leqslant-1$ and $\psi_{n \downarrow}=\tau e^{i k \downarrow n a}$ for $n \geqslant 2$, with $\tau$ and $r$ as the transmission and reflection probability amplitudes. The wave number $k_{\downarrow}$ is related to the total energy $E$ by means of $k_{\downarrow} a$ $=\cos ^{-1}\left[\left(E+\Delta_{Z} / 2\right) /(-2 t)\right]$. For electrons with spin $\uparrow$, their energy outside the Rashba region falls below the band bottom. As a result, we take the wave amplitudes given by $\psi_{n \uparrow}$ $=c e^{k_{\uparrow} n a}$ for $n \leqslant 0$ and $\psi_{n \uparrow}=d e^{-k_{\uparrow} n a}$ for $n \geqslant 1$, where $k_{\uparrow} a$ $=\cosh ^{-1}\left[\left(E-\Delta_{Z} / 2\right) /(-2 t)\right]$. Substituting the total wave function into the Schrödinger equation and projecting over the sites of the Rashba region $\langle n \sigma|$, we find the transmission,

$$
\tau=\left[1+\frac{\lambda^{2}}{t^{2}} \frac{t^{2}\left(1-e^{k_{\uparrow} a+i k_{\downarrow} a}\right)+\left(\lambda^{2}+t^{2}\right) e^{2 i k_{\downarrow} a}}{\left(\lambda^{2}-t^{2}\left(e^{2 k_{\uparrow} a}-1\right)\right)\left(e^{2 i k_{\downarrow} a}-1\right)}\right]^{-1},
$$

which allows to determine the exact condition for the occurrence of zeros in the transmission function $T=|\tau|^{2}$ :

$$
E^{(0)}=\frac{\Delta_{Z}}{2}-2 t \frac{1+\lambda^{2} / 2 t^{2}}{\sqrt{1+\lambda^{2} / t^{2}}} .
$$

This expression has a very appealing form. For a given value of $\lambda$, the antiresonance energy $E^{(0)}$ lies to the left of $-2 t$ $+\frac{\Delta_{z}}{2}$, as shown in the numerical results of Figs. 3 and 4 . Moreover, it predicts that the dip shifts to lower energies as the Rashba interaction strength increases. This is reproduced in Fig. 6, where we plot a characteristic $T$ as a function of energy for $\Delta_{Z}=0.1 t$. In addition, the dip broadens as $\lambda$ increases, in excellent agreement with the numerical results of the continuum model (see Fig. 3). Equation (21) also explains why the critical Zeeman splitting $\Delta_{z}^{\text {crit }}$ below which the dip disappears is so small since the antiresonance is observable only if $E^{(0)}$ lies above the band bottom, i.e., $E^{(0)} \geqslant-2 t$ $-\frac{\Delta_{Z}}{2}$. It then follows that $\Delta_{z}^{\text {crit }}=2 t f(\lambda / t)$, where $f(x)=(1$ $\left.+x^{2} / 2\right) / \sqrt{1+x^{2}}-1$ is a slowly increasing function of $x$ for 0 $\leqslant x \leqslant 1$. As a result, $\Delta_{z}^{\text {crit }} \leq 0.12 t$, which is around 2 orders of magnitude smaller than the bandwidth. Incidentally, we also find an upper bound of the Rashba strength, $\lambda^{\text {crit }}$ $=\sqrt{\Delta_{Z}^{2} / 2+2 \Delta_{Z} t+\left(t+\Delta_{Z} / 2\right) \sqrt{\Delta_{Z}^{2}+4 t \Delta_{Z}}}$, above which the dip vanishes. For $\Delta_{Z}=0.1 t$, we obtain $\lambda^{\text {crit }} \simeq 0.94 t$. This is an interesting feature for applications since very strong Rashba

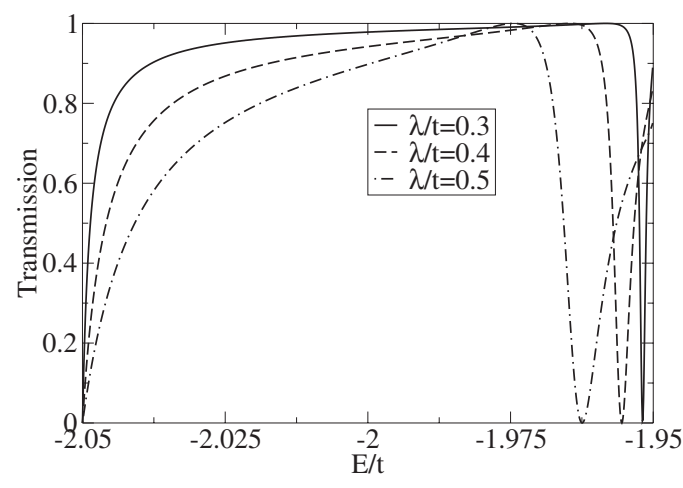

FIG. 6. Transmission versus Fermi energy for $\Delta_{Z}=0.1$ and different spin-orbit strengths. All energies are given in units of $t$.

couplings are not necessary to generate the antiresonance.

The considerations made above suggest that the antiresonance has a Fano line shape $\propto(\epsilon+q)^{2} /\left(\epsilon^{2}+1\right)$, but it is hard to demonstrate with controlled approximations that Eq. (20) has exactly this form. Perhaps it is more instructive to consider a closely related model, in which the spin flip interaction due to the Rashba coupling is restricted to a single point (see Fig. 7). Then, the Hamiltonian reads

$$
\mathcal{H}_{s f}=\sum_{i} s \frac{\Delta_{Z}}{2} c_{i \sigma}^{\dagger} c_{i \sigma}-\sum_{\langle i j\rangle} t\left(c_{i \sigma}^{\dagger} c_{j \sigma}+\text { H.c. }\right)+\lambda c_{0 \uparrow}^{\dagger} c_{0 \downarrow}+\text { H.c. }
$$

As before, the spin-up and spin-down energy bands are shifted by a Zeeman splitting. The coupling between spins at the central site may represent the action of external magnetic field pointing in a direction perpendicular to the spin quantization axis or other source of spin flipping. Spin flip interactions in quantum dots have recently received much attention. ${ }^{57-60}$

We take the wave function ansatz $\psi_{n \downarrow}=e^{i k_{\downarrow} n}+r e^{-i k_{\downarrow} n}$ for $n \leqslant-1$ and $\psi_{n \downarrow}=\tau e^{i k \downarrow^{n}}$ for $n \geqslant 1$ for the propagating states and $\psi_{n \uparrow}=c e^{k_{\uparrow} n}$ for $n \leqslant-1$ and $\psi_{n \uparrow}=d e^{-k_{\uparrow} n}$ for $n \geqslant 1$ for the evanescent states. From the tight-binding equations, we obtain the transmission amplitude,

$$
\tau=\frac{2 i t \sin k_{\downarrow} a}{2 i t \sin k_{\downarrow} a+\frac{\lambda^{2}}{2 t \sinh k_{\uparrow} a}} .
$$

We can define the broadening $\Gamma$, which measures the coupling strength between spin-up and spin-down states and is

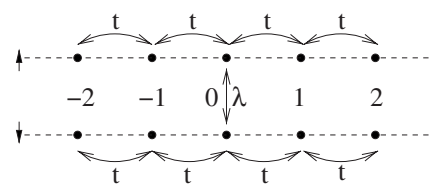

FIG. 7. Sketch of the system considered in the discussion for a pointlike spin-flip interaction between propagating (spin-down) and evanescent (spin-up) states along a one-dimensional site lattice. 
proportional to the density of states (per unit length) for electrons with spin down. It reads

$$
\Gamma=\lambda^{2} \rho_{\downarrow}=\frac{\lambda^{2}}{2 t \sin k_{\downarrow} a} .
$$

Using this result and the expressions for $k_{\uparrow}$ and $k_{\downarrow}$ written above, we find the expression for the transmission probability,

$$
T=|\tau|^{2}=\frac{\left(E-\Delta_{Z} / 2\right)^{2}-(2 t)^{2}}{\left(E-\Delta_{Z} / 2\right)^{2}-(2 t)^{2}+\Gamma^{2}},
$$

valid for energies around the $T=0$ point $E=-2 t+\Delta_{Z} / 2$. We note that Eq. (25) has the desired Fano form. In the conventional Fano effect, the coupling takes place between a bound state immersed in a continuum band and the propagating states. ${ }^{47}$ Here, the role of the bound state is played by the evanescent modes which, due to the spin flip interaction, are coupled to the propagating states with opposite spins.

\section{Angular dependence}

We now discuss the angular dependence of the $\vec{B}$ field direction. In the strict $1 \mathrm{D}$ case, only one mode is needed. Thus, we expand the wave function in the two-spinor basis which, in the case of an in-plane field $\vec{B}$ $=(B \cos \theta, B \sin \theta, 0)$, takes the form

$$
\Psi(x)=\psi_{1}(x) \chi_{+}(\eta)+\psi_{2}(x) \chi_{-}(\eta),
$$

with

$$
\chi_{ \pm}=\frac{1}{\sqrt{2}}\left(\begin{array}{c}
1 \\
\pm e^{i \theta}
\end{array}\right)
$$

the spinors in the $\vec{B}$ direction.

Substituting $\Psi$ in the Schrödinger equation with the Hamiltonian given by Eq. (2), we obtain a pair of coupled equations for $\psi_{1}$ and $\psi_{2}$ :

$$
\begin{aligned}
\psi_{1}^{\prime \prime} & -2 i k_{R} \sin \theta \psi_{1}^{\prime}+\left(k_{F}^{2}-k_{B}^{2}-i k_{R}^{\prime} \sin \theta\right) \psi_{1} \\
& =-\left(2 k_{R} \psi_{2}^{\prime}+k_{R}^{\prime} \psi_{2}\right) \cos \theta, \\
\psi_{2}^{\prime \prime} & +2 i k_{R} \sin \theta \psi_{2}^{\prime}+\left(k_{F}^{2}+k_{B}^{2}+i k_{R}^{\prime} \sin \theta\right) \psi_{2} \\
& =\left(2 k_{R} \psi_{1}^{\prime}+k_{R}^{\prime} \psi_{1}\right) \cos \theta,
\end{aligned}
$$

where primes indicate $d / d x$, and we recall $k_{R}(x)=m \alpha(x) / \hbar^{2}$. We use the following gauge transformation:

$$
\begin{aligned}
\psi_{1} & =\widetilde{\psi}_{1} e^{i \sin \theta \xi(x)}, \\
\psi_{2} & =\widetilde{\psi}_{2} e^{-i \sin \theta \xi(x)},
\end{aligned}
$$

where $\xi(x)=\int^{x} d x^{\prime} k_{R}\left(x^{\prime}\right)$, in order to eliminate the first derivatives in Eqs. (28) and (29), which are transformed into

$$
\left(\begin{array}{ll}
H_{11} & H_{12} \\
H_{21} & H_{22}
\end{array}\right)\left(\begin{array}{l}
\tilde{\psi}_{1} \\
\tilde{\psi}_{2}
\end{array}\right)=E_{F}\left(\begin{array}{l}
\tilde{\psi}_{1} \\
\widetilde{\psi}_{2}
\end{array}\right),
$$

where the elements of the Hamiltonian matrix are

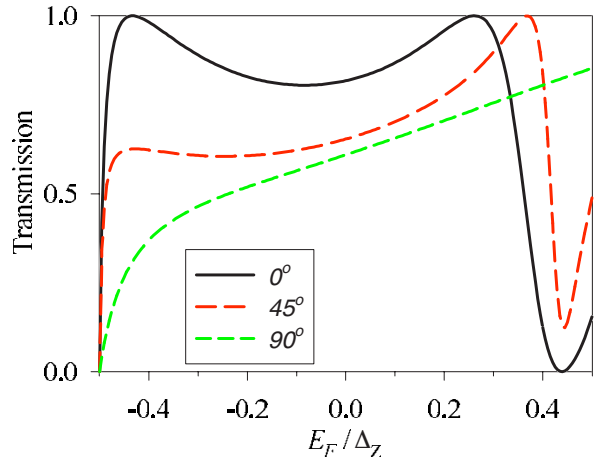

FIG. 8. (Color online) Transmission as a function of Fermi energy varying the orientation of the in-plane magnetic field. The azimuthal angle $\theta$ for each curve is given in the legend. We take $\alpha=6.8 \hbar^{2} / m l$ and $\Delta_{Z}=6.4 \hbar^{2} / m l^{2}$.

$$
H_{11}=\lambda\left(\frac{d^{2}}{d x^{2}}+k_{R}^{2} \sin ^{2} \theta-\frac{m \Delta_{Z}}{\hbar^{2}}\right),
$$

$$
\begin{aligned}
& H_{12}=\lambda \cos \theta e^{-2 i \xi \sin \theta}\left(2 i k_{R}^{2} \sin \theta-k_{R}^{\prime}-2 k_{R} \frac{d}{d x}\right), \\
& H_{21}=\lambda \cos \theta e^{2 i \xi \sin \theta}\left(2 k_{R} \frac{d}{d x}+2 i k_{R}^{2} \sin \theta+k_{R}^{\prime}\right), \\
& H_{22}=\lambda\left(\frac{d^{2}}{d x^{2}}+k_{R}^{2} \sin ^{2} \theta+\frac{m \Delta_{Z}}{\hbar^{2}}\right),
\end{aligned}
$$

with $\lambda=-\hbar^{2} / 2 m$. It is clear that for $\theta=\pi / 2$ the amplitudes $\widetilde{\psi}_{1}$ and $\widetilde{\psi}_{2}$ decouple and for energies inside the gap the transmission is given simply by the propagating state $\tilde{\psi}_{2}$. Then, propagating and evanescent states become decoupled, and no dip is expected. Only for angles away from $\pi / 2$ does the problem become nontrivial since $\widetilde{\psi}_{2}$ couples with the evanescent amplitude $\widetilde{\psi}_{1}$. Numerical results shown in Fig. 8 confirm this expectation.

\section{QUASI-ONE-DIMENSIONAL WIRE}

The preceding section has shown that the Fano resonance phenomenon manifests itself in 1D quantum wires with Zeeman splitting and a local Rashba interaction. Real wires, of course, have always some small extension in the lateral direction. In this section, we analyze the influence of this extra dimension by considering a quasi-1D wire, including the $y$ direction. This lateral $y$ confinement is usually weaker than the vertical $z$ confinement. Thus, we neglect the contribution of the $y$-confining electric field to the Rashba strength (see, however, Ref. 61). For simplicity, we consider a transverse potential of parabolic type, $m \omega_{0}^{2} y^{2} / 2$.

The additional spatial dimension is relevant now because the transverse momentum $p_{y}$ explicitly appears in the Rashba spin-orbit interaction, as shown by Eq. (1). It is also worth stressing that the new term in Eq. (1), proportional to $p_{y} \sigma_{x}$, precludes the use of the analytical solution discussed in Sec. 


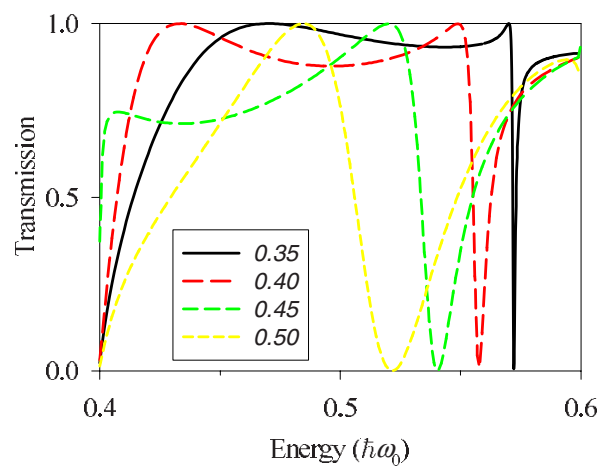

FIG. 9. (Color online) Transmission as a function of the Fermi energy for a quasi-1D wire with transverse parabolic confinement characterized by $\omega_{0}$. A Rashba region of length $l=8 l_{0}$ and a Zeeman energy $\Delta_{Z}=0.2 \hbar \omega_{0}$ with the magnetic field along the wire $(\theta=0)$ have been used. The legend gives the numerical values of $\alpha / \hbar \omega_{0} l_{0}$ for the different curves.

II A for a wire with an extended Rashba interaction. In fact, it is well known that it causes the formation of textured spin states lacking well defined spin quantization axis even for a fixed value of the wave number $k .^{10,26}$ The Rashba coupling $\alpha(x)$ is assumed to be nonzero only in a region of length $l$, where it takes the value $\alpha$, as in Sec. II B. We also include the Zeeman coupling, as in Sec. II A, of a magnetic field oriented along a certain azimuthal angle $\theta$. The full Hamiltonian thus reads

$$
\mathcal{H}_{\mathrm{Q} 1 \mathrm{D}}=\frac{p_{x}^{2}+p_{y}^{2}}{2 m}+\frac{1}{2} m \omega_{0}^{2} y^{2}+\frac{\Delta_{Z}}{2}\left(\cos \theta \sigma_{x}+\sin \theta \sigma_{y}\right)+\mathcal{H}_{R}
$$

A natural unit system for the present quasi-1D model is set by the wire transverse potential, with energy unit $\hbar \omega_{0}$ (oscillator energy) and length unit $l_{0}=\sqrt{\hbar / m \omega_{0}}$ (oscillator length). In what follows, the numerical values for the Rashba region length $l$, spin-orbit intensity $\alpha$, and Zeeman energy $\Delta_{Z}$ will be given in these oscillator units. In order to obtain the transmission of the system modeled by Eq. (37), we have used the quantum transmitting boundary algorithm as in Ref. 30. The Schrödinger equation is discretized on a uniform grid using finite differences for the derivatives and imposing scattering boundary conditions. The reader is referred to Refs. 30 and 62 for additional details of the method.

The existence of the Fano line shapes in a quasi-1D wire, with a transmission zero at a given energy, is clearly shown in Fig. 9. This result proves that the physical effect elucidated with the tight-binding model of Sec. II C is robust and persists in more realistic models. There is also a nice qualitative agreement with the 1D results of Fig. 3. In all three cases (tight binding, 1D, and quasi-1D), increasing the value of $\alpha$ leads to a shift toward lower energies of the transmission zero and to an important broadening of the transmission dip. These are very appealing features related to practical applications in spintronic devices since they could allow to control the transmission by tuning $\alpha$; the device operation

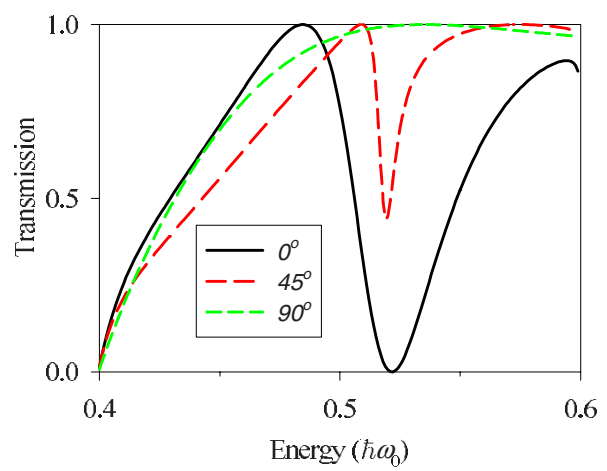

FIG. 10. (Color online) Transmission versus Fermi energy varying the orientation of the in-plane magnetic field. The azimuthal angle $\theta$ for each curve is given in the legend. We use a Rashba region of length $l=8 l_{0}$, Zeeman energy $\Delta_{Z}=0.2 \hbar \omega_{0}$, and spin-orbit coupling strength $\alpha=0.5 \hbar \omega_{0} l_{0}$.

would not be very sensitive to small changes in $\alpha$ due to the broadness of the dip.

The scales used in Fig. 9 are of the same order as in Fig. 3. For example, for a confinement strength $\hbar \omega_{0}=0.1 \mathrm{meV}$ in an InAs wire, we obtain $l \simeq 1.5 \mu \mathrm{m}, \Delta_{Z} \simeq 0.02 \mathrm{meV}(B$ $\simeq 20 \mathrm{mT}$ ), and $\alpha \simeq 9 \mathrm{meV} \mathrm{nm}$.

When the magnetic field is oriented along the wire as in Fig. 9, the interference leading to the Fano profiles in the transmission is maximal. On the contrary, for transverse orientation $\theta=\pi / 2$, it completely disappears (see Fig. 10). This behavior is in agreement with the analysis of Sec. II D in the 1D case, where it was shown that the mixings $H_{12}$ and $H_{21}$ vanish for $\theta=\pi / 2$.

The evolution with the Zeeman field intensity for the quasi-1D case is shown in Fig. 11. The behavior is again qualitatively similar to the $1 \mathrm{D}$ case of Fig. 4, with the dip evolving toward smaller energies when decreasing the value of $\Delta_{Z}$. We also notice that, as predicted by the tight-binding result, even for quite small Zeeman energies there is a dip in the transmission.

Thus far, we have considered abrupt interfaces between the normal sides and the Rashba region. Using the quasi-1D grid calculation, we can also address the influence of the smoothness in the transition of the Rashba coupling strength from zero to the finite value $\alpha$, which is closer to reality. We model each interface using a Fermi function with a diffusivity $d$,

$$
\alpha(x)=\alpha\left[\frac{1}{1+e^{(x-l / 2) / d}}-\frac{1}{1+e^{(x+l / 2) / d}}\right] .
$$

Figure 12 shows the results for different values of $d$. The transmission curve coincides with the abrupt interface limit when $d \sim 0.2 l_{0}$, while for increasing $d$ the interface becomes smoother but the transmission zero remains visible and the dip position does not change much. This is a crucial observation - the transmission zeros we find are roughly independent of the precise profile of the Rashba strength. This robustness has an obvious importance for potential spintronic applications. 


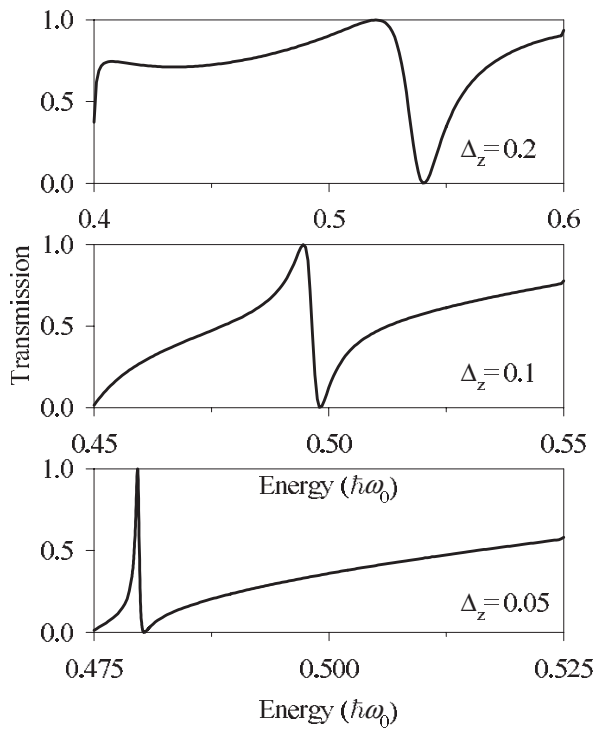

FIG. 11. Transmission as a function of the Fermi energy for a quasi-1D wire with a Rashba region of $l=8 l_{0}$ and a magnetic field along $\theta=0$. The different panels correspond to the given Zeeman energies (in units of $\hbar \omega_{0}$ ). In each panel, solid and dashed lines correspond, respectively, to a value of $\alpha / \hbar \omega_{0} l_{0}$ of 0.45 and 0.40 , respectively.

\section{CONCLUSIONS}

We have performed a theoretical analysis of the transport properties of a ballistic quantum wire with a spatially inhomogeneous Rashba interaction in the presence of an external magnetic field giving rise to Zeeman spin splitting. When the Rashba coupling dominates the magnetic field, an energy pseudogap develops in the wire spectrum. We find abrupt transmission line shapes when the Fermi energy lies within the pseudogap. The line shapes are narrow and asymmetric, and the transmission reaches zero for energies near the gap closing. We have discussed a minimal tight-binding model that reproduces the essential features of the resonances, yielding analytical expressions for the line shape dependence on Fermi energy, Rashba intensity, and Zeeman splitting.

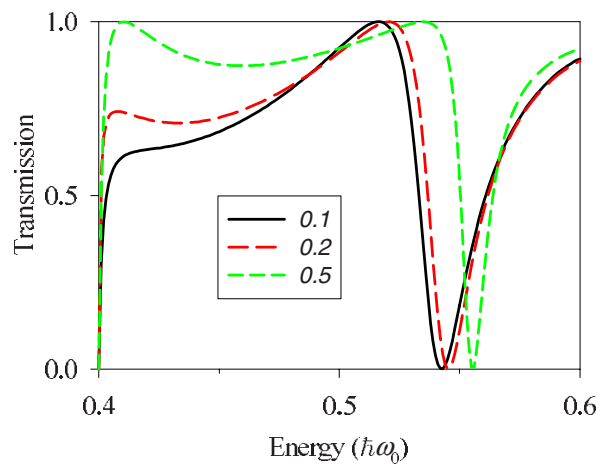

FIG. 12. (Color online) Transmission versus Fermi energy for $\alpha=0.45 \hbar \omega_{0} l_{0}$ and $\Delta_{Z}=0.2 \hbar \omega_{0}$. Each curve corresponds to a different diffusivity $d$, as given in the legend in units of $l_{0}$, for a Rashba region of length $l=8 l_{0}$.
Qualitatively, the evanescent band plays the role of a quasibound state that the confined Rashba interaction couples to the propagating states. The evanescent waves are not true bound states, but when the Fermi energy approaches the evanescent band bottom, electrons scattering off the Rashba region become strongly affected, leading to perfect reflection. Numerical results in realistic quantum wires agree with the purely 1D case. Finally, we have analyzed the behavior of the resonances when the angular orientation of the magnetic field is changed and the interfaces become smoother.

The system studied here could work as a current modulator device. ${ }^{63}$ For slight variations of the Fermi energy, which can be externally controlled, we have shown that the transmission changes dramatically between two limit values (1 and 0 ) across the antiresonance. Our proposal has a number of differences compared to the Datta-Das spin transistor. ${ }^{1}$ First, the latter device modulates the current independently of the energy of the injected electrons since the phase difference $\Delta \theta=2 m \alpha l / \hbar^{2}$ that governs the spin precession is independent of the wave vector. Then, small changes of $\alpha$ or $l$ strongly affects the working points of the transistor, whereas in our case these points are not very sensitive to small variations of the external parameters such as the external magnetic field, the Rashba strength, or the interface diffusivity. Moreover, a 100\% current modulation is hard to achieve in the Datta-Das transistor, especially when intersubband coupling is taken into account, whereas in our case the modulation is rather abrupt and is preserved even when adjacent subbands are coupled, an effect which is unavoidable in real quantum wires. We have reported results for the lowest spinsplit subband but have checked that similar pronounced dips are seen in higher subbands. Our results differ also with those of Ref. 30 since in that case the antiresonances reached zero only after a fine tuning of the parameters. Here, our only requirement is that the Fermi energy should lie within the spectrum pseudogap.

As far as the discussion in 1D wires is concerned, the field directions orthogonal to the Rashba field (along $y$, according to the parametrization of the Hamiltonian we have employed) are equivalent. However, in the quasi-1D case, one of these directions (the one perpendicular to both the Rashba field and the electron propagation) induces orbital effects. Here, we have restricted ourselves to fields giving rise only to Zeeman splittings since the study of orbital effects in inhomogeneous systems requires knowledge of the evanescent states when the magnetic field is applied perpendicular to the wire plane. This is not a trivial task, and it seems to be a promising avenue of future research. Reference 25 finds important changes in the spectrum structure of a quantum wire with uniform Rashba interaction and perpendicular magnetic fields. However, our sharp antiresonances show up even in the presence of rather small Zeeman splittings. Therefore, we expect that the dips should still be visible even when orbital effects are taken into account, provided the magnetic field length is much larger than the confinement length.

In our discussion, we have neglected electron-electron interaction effects, which may lead to Luttinger liquid effects in 1D ballistic wires when the interactions are screened like in a wire with electric-field induced spin-orbit interactions. ${ }^{64,65}$ When Zeeman splittings are present ${ }^{66,67}$ the 
transmission seems to be altered by electron-electron interactions, although these works neglect the intersubband coupling term of the Rashba interaction. In fact, for ballistic wires without Rashba coupling but multiple populated subbands, a simple mean-field approach demonstrates ${ }^{68}$ that Coulomb interactions are crucial to an understanding of rectification effects observed in nanojunction rectifiers. ${ }^{69}$ On the other hand, single-particle effects are shown ${ }^{53}$ to lead to Coulomb blockade antiresonances of the Fano form. Hence, further work is needed to clarify the influence of Coulomb interactions in the conductance of a quantum wire with Zeeman splitting and a localized Rashba interaction.

Finally, we would like to comment on the role of disorder. Our findings have a quantum interference origin and are not less robust than any other coherent interference effect commonly found in mesoscopic systems. Since samples with suf- ficiently long coherence lengths and mean free path are currently available, ${ }^{70,71}$ we expect that the effects we discuss will be robust against weak disorder. The relevance of impurity scattering in the generation of pure spin currents has been recently shown. ${ }^{72,73}$ Nevertheless, in this work, we analyze charge currents, which are much more robust against sample imperfections. This could be the subject of future work, in which the impact of disorder in the transmission modulation should be carefully investigated.

\section{ACKNOWLEDGMENTS}

This work was supported by the Spanish MEC Grant No. FIS2005-02796, the "Ramón y Cajal" program and the SRC/ERC program (R11-2000-071).
${ }^{1}$ S. Datta and B. Das, Appl. Phys. Lett. 56, 665 (1990).

${ }^{2}$ E. I. Rashba, Fiz. Tverd. Tela (Leningrad) 2, 1224 (1960) [Sov. Phys. Solid State 2, 1109 (1960)].

${ }^{3}$ Y. Bychkov and E. I. Rashba, J. Phys. C 17, 6039 (1984).

${ }^{4}$ Y. Sato, S. Gozu, T. Kikutani, and S. Yamada, Physica B 272, 114 (1999).

${ }^{5}$ A. V. Moroz and C. H. W. Barnes, Phys. Rev. B 60, 14272 (1999).

${ }^{6}$ F. Mireles and G. Kirczenow, Phys. Rev. B 64, 024426 (2001).

${ }^{7}$ A. A. Kiselev and K. W. Kim, Appl. Phys. Lett. 78, 775 (2001).

${ }^{8}$ L. W. Molenkamp, G. Schmidt, and G. E. W. Bauer, Phys. Rev. B 64, 121202(R) (2001).

${ }^{9}$ J. C. Egues, G. Burkard, and D. Loss, Phys. Rev. Lett. 89, 176401 (2002); J. C. Egues, G. Burkard, D. S. Saraga, J. Schliemann, and D. Loss, Phys. Rev. B 72, 235326 (2005).

${ }^{10}$ M. Governale and U. Zülicke, Phys. Rev. B 66, 073311 (2002).

${ }^{11}$ G. Feve, W. D. Oliver, M. Aranzana, and Y. Yamamoto, Phys. Rev. B 66, 155328 (2002).

${ }^{12}$ E. N. Bulgakov and A. F. Sadreev, Phys. Rev. B 66, 075331 (2002).

${ }^{13}$ E. A. de Andrada e Silva and G. C. La Rocca, Phys. Rev. B 67, 165318 (2003).

${ }^{14}$ P. Streda and P. Seba, Phys. Rev. Lett. 90, 256601 (2003).

${ }^{15}$ Th. Schäpers, J. Knobbe, and V. A. Guzenko, Phys. Rev. B 69, 235323 (2004).

${ }^{16}$ Yu. V. Pershin, J. A. Nesteroff, and V. Privman, Phys. Rev. B 69, 121306(R) (2004).

${ }^{17}$ J. A. Nesteroff, Yu. V. Pershin, and V. Privman, Phys. Rev. Lett. 93, 126601 (2004).

${ }^{18}$ M. Cahay and S. Bandyopadhyay, Phys. Rev. B 69, 045303 (2004).

${ }^{19}$ X. F. Wang, Phys. Rev. B 69, 035302 (2004).

${ }^{20}$ I. A. Shelykh and N. G. Galkin, Phys. Rev. B 70, 205328 (2004).

${ }^{21}$ F. Zhai and H. Q. Xu, Phys. Rev. Lett. 94, 246601 (2005).

${ }^{22}$ J. Knobbe and Th. Schäpers, Phys. Rev. B 71, 035311 (2005).

${ }^{23}$ R. G. Pereira and E. Miranda, Phys. Rev. B 71, 085318 (2005).

${ }^{24}$ C. L. Romano, S. E. Ulloa, and P. I. Tamborenea, Phys. Rev. B 71, 035336 (2005).

${ }^{25}$ S. Debald and B. Kramer, Phys. Rev. B 71, 115322 (2005).
${ }^{26}$ Ll. Serra, D. Sánchez, and R. López, Phys. Rev. B 72, 235309 (2005)

${ }^{27}$ L. Zhang, P. Brusheim, and H. Q. Xu, Phys. Rev. B 72, 045347 (2005).

${ }^{28}$ S. Zhang, R. Liang, E. Zhang, L. Zhang, and Y. Liu, Phys. Rev. B 73, 155316 (2006).

${ }^{29}$ A. Reynoso, G. Usaj, and C. A. Balseiro, Phys. Rev. B 73, 115342 (2006).

${ }^{30}$ D. Sánchez and Ll. Serra, Phys. Rev. B 74, 153313 (2006).

${ }^{31}$ J.-S. Jeong and H.-W. Lee, Phys. Rev. B 74, 195311 (2006).

${ }^{32}$ Th. Schapers, V. A. Guzenko, M. G. Pala, U. Zülicke, M. Governale, J. Knobbe, and H. Hardtdegen, Phys. Rev. B 74, 081301(R) (2006)

${ }^{33}$ L. Zhang, F. Zhai, and H. Q. Xu, Phys. Rev. B 74, 195332 (2006).

${ }^{34}$ C. A. Perroni, D. Bercioux, V. Marigliano Ramaglia, and V. Cataudella, J. Phys.: Condens. Matter 19, 186227 (2007).

${ }^{35}$ A. H. Nevidomskyy and K. Le Hur, arXiv:cond-mat/0608340 (unpublished).

${ }^{36}$ J. Nitta, T. Akazaki, H. Takayanagi, and T. Enoki, Phys. Rev. Lett. 78, 1335 (1997).

${ }^{37}$ G. Engels, J. Lange, Th. Schäpers, and H. Lüth, Phys. Rev. B 55, R1958 (1997).

${ }^{38}$ D. Grundler, Phys. Rev. Lett. 84, 6074 (2000).

${ }^{39}$ B. J. van Wees, H. van Houten, C. W. J. Beenakker, J. G. Williamson, L. P. Kouwenhoven, D. van der Marel, and C. T. Foxon, Phys. Rev. Lett. 60, 848 (1988).

${ }^{40}$ D. A. Wharam, T. J. Thornton, R. Newbury, M. Pepper, H. Ritchie, and G. A. C. Jones, J. Phys. C 21, L209 (1988).

${ }^{41}$ C. S. Chu and R. S. Sorbello, Phys. Rev. B 40, 5941 (1989).

${ }^{42}$ P. F. Bagwell, Phys. Rev. B 41, 10354 (1990).

${ }^{43}$ J. Faist, P. Guéret, and H. Rothuizen, Phys. Rev. B 42, R3217 (1990).

${ }^{44}$ E. Tekman and S. Ciraci, Phys. Rev. B 43, 7145 (1991).

${ }^{45}$ S. A. Gurvitz and Y. B. Levinson, Phys. Rev. B 47, 10578 (1993).

${ }^{46}$ J. U. Nöckel and A. D. Stone, Phys. Rev. B 50, 17415 (1994).

${ }^{47}$ U. Fano, Phys. Rev. 124, 1866 (1961).

${ }^{48}$ F. Cerdeira, T. A. Fjeldly, and M. Cardona, Phys. Rev. B 8, 4734 (1973). 
${ }^{49}$ J. Göres, D. Goldhaber-Gordon, S. Heemeyer, M. A. Kastner, H. Shtrikman, D. Mahalu, and U. Meirav, Phys. Rev. B 62, 2188 (2000).

${ }^{50}$ K. Kobayashi, H. Aikawa, S. Katsumoto, and Y. Iye, Phys. Rev. Lett. 88, 256806 (2002).

${ }^{51}$ M. Valín-Rodríguez, A. Puente, and Ll. Serra, Phys. Rev. B 69, 085306 (2004).

${ }^{52}$ J. Cserti, A. Csordás, and U. Zülicke, Phys. Rev. B 70, 233307 (2004).

${ }^{53}$ R. López, D. Sánchez, and Ll. Serra, Phys. Rev. B 76, 035307 (2007).

${ }^{54}$ Ll. Serra, D. Sánchez, and R. López, Phys. Rev. B 76, 045339 (2007).

${ }^{55}$ E. N. Bulgakov, K. N. Pichugin, A. F. Sadreev, P. Streda, and P. Seba, Phys. Rev. Lett. 83, 376 (1999).

${ }^{56}$ T. Ando, Phys. Rev. B 40, 5325 (1989).

${ }^{57}$ W. Rudzinski and J. Barnas, Phys. Rev. B 64, 085318 (2001).

${ }^{58}$ R. López and D. Sánchez, Phys. Rev. Lett. 90, 116602 (2003).

${ }^{59}$ M.-S. Choi, D. Sánchez, and R. López, Phys. Rev. Lett. 92, 056601 (2004).

${ }^{60}$ B. Dong, G. H. Ding, H. L. Cui, and X. L. Lei, Europhys. Lett. 69, 424 (2005).

${ }^{61}$ Y. Jiang and L. Hu, Phys. Rev. B 74, 075302 (2006).
${ }^{62}$ C. S. Lent and D. J. Kirkner, J. Appl. Phys. 67, 6353 (1990).

${ }^{63}$ Very recently, it is suggested in J. Wan, M. Cahay, and S. Bandyopadhyay, J. Appl. Phys. 102, 034301 (2007) that a similar system could work as a sensor of tiny magnetic fields and as a digital binary switch.

${ }^{64}$ W. Häusler, Phys. Rev. B 63, 121310(R) (2001).

${ }^{65}$ V. Gritsev, G. I. Japaridze, M. Pletyukhov, and D. Baeriswyl, Phys. Rev. Lett. 94, 137207 (2005).

${ }^{66}$ H. C. Lee and S.-R. Eric Yang, Phys. Rev. B 72, 245338 (2005).

${ }^{67}$ P. Devillard, A. Crepieux, K. I. Imura, and T. Martin, Phys. Rev. B 72, 041309(R) (2005).

${ }^{68}$ M. Büttiker and D. Sánchez, Phys. Rev. Lett. 90, 119701 (2003).

${ }^{69}$ A. M. Song, A. Lorke, A. Kriele, J. P. Kotthaus, W. Wegscheider, and M. Bichler, Phys. Rev. Lett. 80, 3831 (1998).

${ }^{70}$ X. Lou, C. Adelmann, S. A. Crooker, E. S. Garlid, J. Zhang, K. S. Madhukar Reddy, S. D. Flexner, C. J. Palmstron, and P. A. Crowell, Nat. Phys. 3, 197 (2007).

${ }^{71}$ H. C. Koo, H. Yi, J.-B. Ko, J. Chang, S.-H. Han, D. Jung, S.-G. Huh, and J. Eom, Appl. Phys. Lett. 90, 022101 (2007).

${ }^{72}$ J. I. Inoue, G. E. W. Bauer, and L. W. Molenkamp, Phys. Rev. B 70, 041303(R) (2004).

${ }^{73}$ M. W. Wu and J. Zhou, Phys. Rev. B 72, 115333 (2005). 